\title{
Prehabilitation in lung cancer resection-are we any closer to the ideal program?
}

\author{
Kajan Mahendran ${ }^{1}$, Babu Naidu ${ }^{2}$ \\ ${ }^{1}$ Department of Thoracic Surgery, Birmingham Heartlands Hospital, Birmingham, UK; ${ }^{2}$ Institute of Inflammation and Ageing, College of Medical \\ and Dental Sciences, University of Birmingham, Birmingham, UK \\ Correspondence to: Babu Naidu. Department of Thoracic Surgery Research, Heartlands Hospital, Bordesley Green East, Birmingham B9 5SS, UK. \\ Email: b.naidu@bham.ac.uk. \\ Provenance and Peer Review: This article was commissioned and reviewed by the Section Editor Laura Chiara Guglielmetti (Cantonal Hospital \\ Winterthur, Kantonsspital Winterthur, Switzerland). \\ Comment on: Liu Z, Qiu T, Pei L, et al. Two-Week Multimodal Prehabilitation Program Improves Perioperative Functional Capability in Patients \\ Undergoing Thoracoscopic Lobectomy for Lung Cancer: A Randomized Controlled Trial. Anesth Analg 2019. [Epub ahead of print]. \\ Laurent H, Aubreton S, Galvaing G, et al. Preoperative respiratory muscle endurance training improves ventilatory capacity and prevents pulmonary \\ postoperative complications after lung surgery: a randomized controlled trial. Eur J Phys Rehabil Med 2020;56:73-81.
}

Submitted Dec 22, 2019. Accepted for publication Jan 13, 2020.

doi: $10.21037 /$ jtd.2020.02.15

View this article at: http://dx.doi.org/10.21037/jtd.2020.02.15

\section{Introduction}

The drive to increase lung cancer resection rates continues and with it, the need to reduce the perioperative risks and improve post-operative quality of life for an increasingly high-risk cohort of patients. Post-operative pulmonary complications are common and are associated with poorer long-term outcomes (1). The recent ERAS guidelines highlight the importance of preoperative rehabilitation, or prehabilitation, in reducing hospital length of stay and postoperative pulmonary complications (2). However, they note the paucity of high-quality evidence in how best to employ it.

We have previously interrogated the evidence for prehabilitation in an attempt to answer the key questions on how to run the ideal programme (3). We read with interest two recently published randomised controlled trials on the topic to see if they can provide evidence to clarify the answers to some of these.

Laurent $e t a l$. performed an open label randomised control trial in 26 patients to evaluate the effect of preoperative respiratory muscle endurance training with change in minute volume achievable and endurance time, a kind of "bleep test" for breathing, as the primary outcome (4). Their patients all underwent anatomical resection for lung cancer at the end of the 3 weeks.

Liu et al. report on a single blind randomised controlled trial of 73 patients undergoing a multimodality rehabilitation program for 2 weeks prior to lung cancer resection (5).

\section{Candidates}

While putatively all patients would benefit from some prehabilitation, higher risk patients seem to show the most benefit.

Liu et al. present a cohort of relatively young and fit patients with the selection criteria including only those under the age of 70 and excluding those with certain comorbid conditions or if the ASA was $>3$. The French study cohort is also relatively young (mean age 64) but with a high number (almost 50\% in both groups) of patents having surgery post neoadjuvant chemotherapy. There is no data provided on comorbidities.

There are a number of studies performed which suggest a broad spectrum of patients may benefit from prehabilitation. Again, there is no consensus. In an ideal world where resources are unlimited all patients would be 
sent for the same optimal rehabilitation programme. In reality it would be useful to stratify the patients who would benefit the most from the highest intervention. That data is not yet available.

\section{Duration}

The timing of prehabilitation is problematic. Intuitively the longer a subject train, the more fit they become. National Guidelines in the UK are pushing for shorter lung cancer pathways on the grounds that delays to treatment can deleteriously affect survival (6). However, the data is incoherent and the real effect of short-term treatment delay is incompletely understood (7).

Liu et al. show that an intensive multimodal program can be as short as 2 weeks and still achieve significant changes in measurable exercise parameters. Laurent et al. have a similarly short program of 3 weeks with only inspiratory muscle training producing a significant difference between groups in measures of respiratory function.

In clinical practice, decision making is straightforward in patients with small peripheral tumours who present with high risk medical histories and can therefore afford to undergo prolonged rehabilitation and also in fit patients with large central tumours for whom no delay can be afforded. It is the patients in between that will pose a challenge to the surgeon in picking the right time to intervene. As the pathways aim to shorten the time to treatment for patients so they should incorporate prehabilitation from the early referral stage to ensure maximal benefit is gleaned. Even if patients don't then go on to have surgery it may provide benefits (8).

\section{Type of training}

There is no consensus on the type of training that should constitute a prehabilitation programme-most studies incorporate some combination of aerobic exercise, strength training, inspiratory muscle training or breathing exercises (3). Within these subheadings there is a great deal of variation and overlap.

Liu et al. have not attempted to differentiate instead incorporating aerobic exercise, strength training and respiratory/inspiratory muscle exercises into their study combined with nutritional advice and meditation for the treatment group. This, they compared to routine clinical care which included smoking cessation in both groups.
They check intensity achieved with exercise with the widely employed Borg scale of perceived exertion and a heart rate monitor. Through this they achieve an increase in preoperative walking distance of $45 \mathrm{~m}$ (mean baseline distance $565 \mathrm{~m}$ ) in a short time frame. This is greater than the reported minimal clinically important distance (14-30.5 $m$ ) in a number of studies (9).

Laurent et al. are much more specific-looking at a particular inspiratory muscle training techniquehyperventilation. To prevent hypocapnia during the training patient's breath through complex equipment attached to a rebreathing bag bespoke to each patient's breathing volumes. The baseline therapy, common to both groups, is usual chest physiotherapy only which included airway clearance techniques and deep breathing exercises. Interestingly with this therapy alone they achieve significant increases in patient's minute volume and hyperventilation endurance time.

A well planned programme should contain a combination of physiotherapy techniques much like the Chinese study. Laurent et al. aim to show the additive benefit of a particular technique. We feel that the ideal prehabilitation should be standardised such that it is easily reproducible not only between centres but most importantly for all patients to complete. There should, however, be a degree of flexibility to allow the programme to fit the patients' needs and drivers to exercise to ensure high compliance and efficacy.

\section{Location}

Hospital based programmes allow for the constant monitoring of adherence to the program and recommended intensity levels because of the presence of expert therapists. While this is suitable in the research setting to ensure compliance, in reality not all patients can access this, either due to a lack of system or personal resources.

Both the studies here utilise a mainly home based prehabilitation programme with initial sessions by a physiotherapist to train them in the exercises required and a method (diary + phone call or weekly visit) to ensure compliance. This is a crucial step in delivering an efficient but effective program. Advancing technology means that patients can have safe and effective prehabilitation programmes in their own homes. Our own institution has designed and tested an App based fitness program with a bluetooth pulse oximeter to check intensity and compliance. So far patient compliance and feedback has 
been encouraging but impact on clinical outcomes has not been tested (10). The ideal programme will be mainly home based but with a high degree of supervision for the patients that will benefit from it.

\section{Discussion}

The search for the ideal predictor of patients with high risk complications continues-it should be easily measurable, an accurate predictor and ideally modifiable. Spirometry and Transfer factor have been validated and constitute a key part of selection criteria in the guidelines, exercise testing in the form of $\mathrm{VO}_{2}$ max measurement is limited to high risk patients only. One of the main design limits particular to Liu et al.'s work is the use of the 6-minute walk distance as an outcome measure. It has conflicting supporting evidence and certainly has not been validated as a risk stratification tool in all patients-for this reason international guidelines have recommended against its routine inclusion in preoperative testing (11). Therefore, the positive conclusion of the study, based on a significant difference between groups in mean walking distance of $60.9 \mathrm{~m}$ perioperatively, is difficult to interpret given that there is no reported minimal clinically important difference reported for patients undergoing lung cancer surgery in the literature. The authors would have been better served powering the study to assess for a significant reduction in post-operative complications or quality of life (which was not shown) or using a more validated tool such as $\mathrm{VO}_{2}$ max.

Laurent et al. report a significant reduction in pulmonary complications but their study is not powered for this. Indeed, when comparing $\mathrm{VO}_{2}$ max, which has been shown to be highly predictive of pulmonary complications after surgery, there was no significant difference between the groups pre and post training. In fact, the $\mathrm{VO}_{2} \max$ in the control group was lower (not significant) than the treatment group pre training, though this small difference alone is unlikely to explain the reduction in pulmonary complications in the latter. The authors do note that the pulmonary complication rate is disproportionately high $(83 \%)$ in the control group without providing a cause.

\section{Conclusions}

While the aims of the studies, to show that short-term prehabilitation which doesn't delay surgery remains beneficial to patients, flaws within the design of both limit the applicability of the studies to clinical practice.
While powering a study large enough to find significant improvement in post-operative pulmonary complications or long-term quality of life may be challenging, the primary outcome measured must at least be a validated surrogate such as $\mathrm{VO}_{2}$ max.

Prehabilitation is firmly embedded in the ERAS guidelines as we strive to improve patients' post-operative outcomes. There remain, however, questions as to how to most efficaciously employ it.

\section{Acknowledgments}

Funding: None.

\section{Footnote}

Conflicts of Interest: All authors have completed the ICMJE uniform disclosure form (available at http://dx.doi. org/10.21037/jtd.2020.02.15). The authors have no conflicts of interest to declare.

Ethical Statement: The authors are accountable for all aspects of the work in ensuring that questions related to the accuracy or integrity of any part of the work are appropriately investigated and resolved.

Open Access Statement: This is an Open Access article distributed in accordance with the Creative Commons Attribution-NonCommercial-NoDerivs 4.0 International License (CC BY-NC-ND 4.0), which permits the noncommercial replication and distribution of the article with the strict proviso that no changes or edits are made and the original work is properly cited (including links to both the formal publication through the relevant DOI and the license). See: https://creativecommons.org/licenses/by-nc-nd/4.0/.

\section{References}

1. Lugg ST, Agostini PJ, Tikka T, et al. Long-term impact of developing a postoperative pulmonary complication after lung surgery. Thorax 2016;71:171-6.

2. Batchelor TJP, Rasburn NJ, Abdelnour-Berchtold E, et al. Guidelines for enhanced recovery after lung surgery: recommendations of the Enhanced Recovery After Surgery (ERAS $\left.{ }^{\circledR}\right)$ Society and the European Society of Thoracic Surgeons (ESTS). Eur J Cardiothorac Surg 2019;55:91-115.

3. Mahendran K, Naidu B. The key questions in 
rehabilitation in thoracic surgery. J Thorac Dis 2018;10:S924-30.

4. Laurent H, Aubreton S, Galvaing G, et al. Preoperative respiratory muscle endurance training improves ventilatory capacity and prevents pulmonary postoperative complications after lung surgery: a randomized controlled trial. Eur J Phys Rehabil Med 2020;56:73-81.

5. Liu Z, Qiu T, Pei L, et al. Two-Week Multimodal Prehabilitation Program Improves Perioperative Functional Capability in Patients Undergoing Thoracoscopic Lobectomy for Lung Cancer: A Randomized Controlled Trial. Anesth Analg 2019. [Epub ahead of print].

6. National Optimal Lung Cancer Pathway Implementation Guide produced by The Lung Clinical Expert Group, Date of issue: August 2017. Available online: http://content. smallerearthtech.co.uk/system/file_uploads/16086/original/ National_Optimal_LUNG_Pathway_Aug_2017.pdf

7. Abrao FC, de Abreu IRLB, Rocha RO, et al. Interaction

Cite this article as: Mahendran K, Naidu B. Prehabilitation in lung cancer resection-are we any closer to the ideal program? J Thorac Dis 2020;12(4):1628-1631. doi: 10.21037/jtd.2020.02.15 between treatment delivery delay and stage on the mortality from non-small cell lung cancer. J Thorac Dis 2018;10:2813-9.

8. Rivas-Perez H, Nana-Sinkam P. Integrating pulmonary rehabilitation into the multidisciplinary management of lung cancer: a review. Respir Med 2015;109:437-42.

9. Bohannon RW, Crouch R. Minimal clinically important difference for change in 6-minute walk test distance of adults with pathology: a systematic review. J Eval Clin Pract 2017;23:377-81.

10. Kadiri SB, Kerr AP, Oswald NK, et al. Fit 4 surgery, a bespoke app with biofeedback delivers rehabilitation at home before and after elective lung resection. J Cardiothorac Surg 2019;14:132.

11. Brunelli A, Charloux A, Bolliger CT et al. ERS/ESTS clinical guidelines on fitness for radical therapy in lung cancer patients (surgery and chemo-radiotherapy). Eur Respir J 2009;34:17-41. 